\title{
Experimental Investigation of Pedestrian Dynamics in Circle Antipode Experiments
}

\author{
Yao Xiao, Rui Jiang, Ziyou Gao, Xingang Li, Yunchao Qu \\ School of Traffic and Transportation, Beijing Jiaotong University, \\ Beijing, China \\ xiaoy@bjtu.edu.cn
}

\begin{abstract}
To explore the pedestrian motion navigation and conflict reaction mechanisms in practice, we organized a series of circle antipode experiments. In the experiments, pedestrians are uniformly initialized on the circle and required to leave for their antipodal positions simultaneously. On the one hand, a conflicting area is naturally formulated in the center region due to the converged shortest routes, so the practical conflict avoidance behaviors can be fully explored. On the other hand, the symmetric experimental conditions of pedestrians, e.g., symmetric starting points, symmetric destination points, and symmetric surroundings, lay the foundation for further quantitative comparisons among participants. The pedestrian trajectories in the experiments are recognized and rotated, and several aspects, e.g., the trajectory space distribution, route length, travel time, velocity distribution, and time-series, are investigated. It is found that: (1) Pedestrians prefer the right-hand side during the experiments; (2) The route length follows a log-normal distribution, the route potential obeys an exponential distribution, and travel time as well as speed are normally distributed; (3) Taking the short routes unexpectedly cost pedestrians plenty of travel time, while detours seem to be time-saving.

Keywords: Circle antipode experiment, pedestrian trajectories, experiment analysis, pedestrian dynamics
\end{abstract}

\section{Introduction}

The circle antipode experiment has been applied as a simulation scene [1-3], but very limited attention is paid to the performance of pedestrians in reality. In the circle antipode experiments, pedestrians are uniformly initialized on a circle, and they are required to reach the antipodal positions. The first significant feature of the experiment is that the shortest routes intersect at the center of the circle, and a crowded area is generally formulated in the center zone. In this case, a pedestrian has to deal with the conflicts with other pedestrians and pass through the crowded area in front. The ability to deal with the situations of conflicts and congestions is a fundamental and core problem in pedestrian research, and here the practical handling ability of conflicts and collisions could be revealed. Another significant but rarely mentioned feature is that each pedestrian has a symmetric initial position and a symmetric destination position in the experiment. In other words, except for the heterogeneity of pedestrians themselves, each pedestrian faces a symmetric experimental condition. Based on it, the results of pedestrian trajectories could be used to maximum possible extent, and more quantitative investigations are possible. Finally, with the development of the video recognition technology and other trajectory recognition technologies in recent years, obtaining the precise trajectories of pedestrians even in crowded situations is not so difficult as before. The achievement of the precise pedestrian trajectories provides more detailed characteristics of microscopic pedestrian behaviors. In summary, the access to the trajectories of the circle antipode experiments provides more possibilities for quantitatively investigating the performance of pedestrians in a challenging conflicting situation.

\section{Experiments}

Up to 64 participants ( 31 females, 33 males) were involved in the experiment, and they were students from the university and aged 18 to 28 . The experiments were conducted on two circles of radii of $5 \mathrm{~m}$ and 10 $\mathrm{m}$, respectively (Figure 1). For each circle, experiments with four pedestrian counts $(8,16,32,64)$ were carried out, each of which was repeated four times. With these specific number of participants (e.g., 
$8=64 / 2^{3}$ ), it is convenient to formulate a symmetric experimental situation for all the participants based on the existing 64 surface marks in practice. In addition, more videos about the experiments can be found on our website, http://pedynamic.com/circle-antipode-experiments/ .

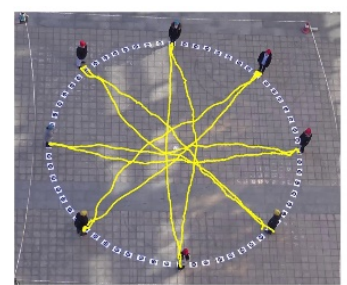

(a)

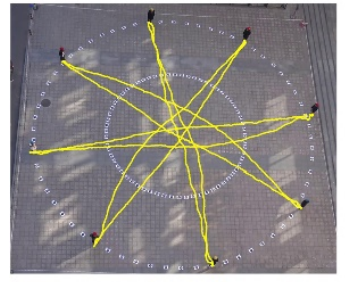

(e)

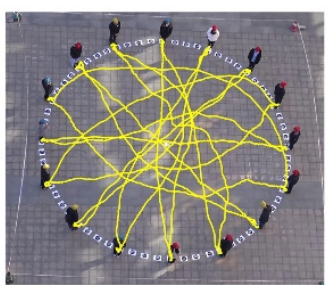

(b)

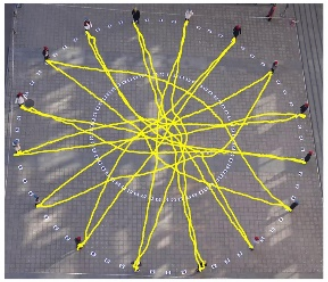

(f)

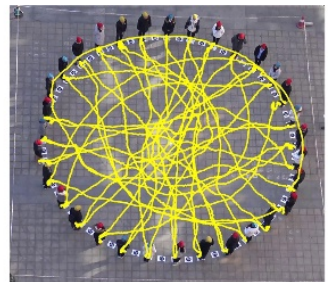

(c)

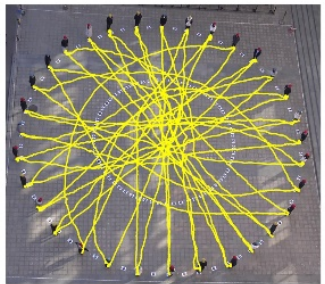

(g)

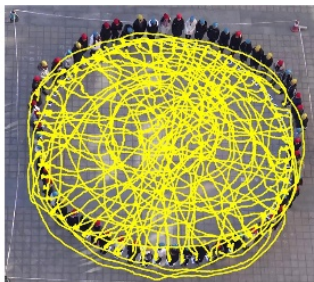

(d)

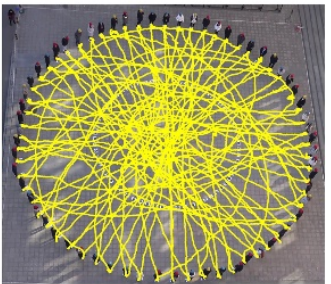

(h)

Figure 1. Original trajectories in the circle experiments. The yellow curves represent the trajectories on the ground. (a)-(d) 8, 16, 32, 64 pedestrians in the circle of 5m. (e)-(h) 8, 16, 32, 64 pedestrians in the circle of $10 \mathrm{~m}$.

\section{Results}

To make the most of the specific symmetric distribution features, the original route trajectories are respectively rotated around the center of the circle until the starting point of the route and the left end of the circle are overlapped. Since the destination of each pedestrian locates at the antipodal position of the starting point, the destination should also be consistent.

\subsection{Side Preference}

Though investigation on the spatial distribution of trajectories, it is found that the probabilities of trajectories in the right side semi-circle are greater than those in the left side semi-circle (Figure 2). That is to say, pedestrians prefer to detour from the right side in our experiments. Past studies [4-5] suggested that the side preference could be interpreted as a cultural bias, and the right side preference in China agrees with our experimental results. What's more, the circle antipode experiments can be regarded as quantitative measurement for side preference.

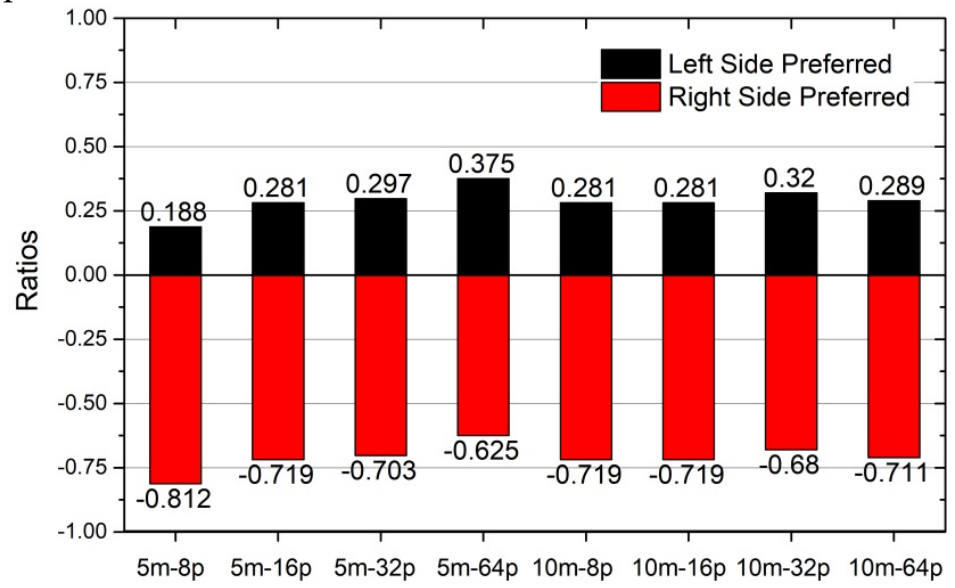

Figure 2. Trajectory distribution in left/right side semicircle 

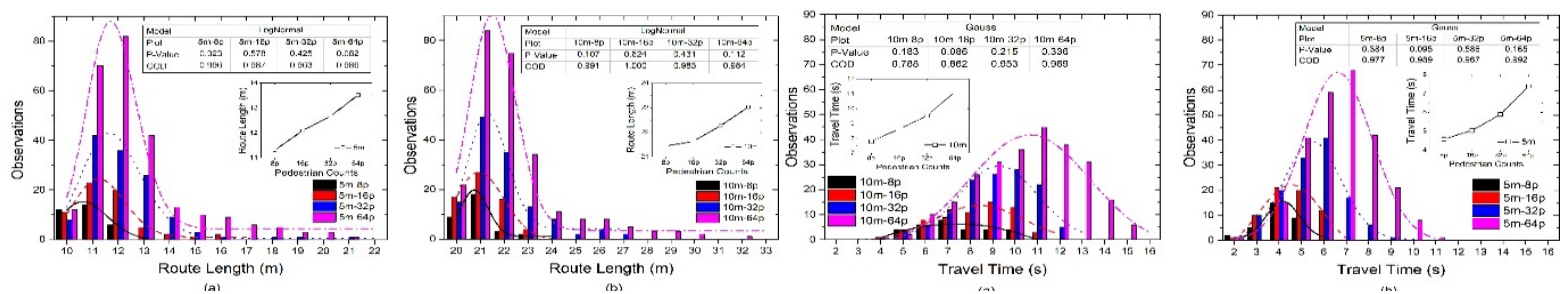

Figure 3. Distributions of the route length and travel time. (a) $5 \mathrm{~m}$ experiments. (b) $10 \mathrm{~m}$ experiments.

\subsection{Distribution of route length and travel time}

The mean values and distributions of the route length are illustrated in Figure 3. The route lengths cover from $10 \mathrm{~m}$ to $21 \mathrm{~m}$ in the $5 \mathrm{~m}$ experiments, while from $20 \mathrm{~m}$ to $33 \mathrm{~m}$ in the $10 \mathrm{~m}$ experiments. On the whole, there is a peak value in each distribution, and the peak value generally exists at the left side of the data range and a long tail exists at the right side. Considering the special shape of the route length distribution, the log-normal distribution is introduced to fit it. In the hypothesis test, the null hypothesis is that the route length distribution follows a log-normal distribution, and the significant level is set as $5 \backslash \%$. The results of the non-parametric test show that the p-values in all experiments are greater than 0.05 (see Figure 4), so the null hypothesis cannot be rejected and the route length obeys a log-normal distribution. Besides, the coefficients of determination (COD) further prove the goodness of fitting.

The mean values and distributions of travel time are illustrated in Figure 3. In the experiments, the mean travel time increases with increasing pedestrian counts. The growing pedestrian counts make the circle more crowded and more pedestrians have to detour or slow down, hence the travel time increases. The travel times cover from 2 seconds to 11 seconds in the $5 \mathrm{~m}$ experiments, while from 3 seconds to 16 seconds in the $10 \mathrm{~m}$ experiments. Each distribution has a peak value which increases with increasing pedestrian counts. The normal (Gauss) distribution is introduced to fit it. The normality tests (Figure 5) and the COD prove that the travel time distribution follows a normal distribution.

\section{References}

[1] Van den Berg J, Lin M, Manocha D. Reciprocal velocity obstacles for real-time multi-agent navigation., IEEE International Conference on Robotics and Automation; 2008: 1928-1935.

[2] Ondřej J, Pettré J, Olivier A-H, Donikian S. A synthetic-vision based steering approach for crowd simulation. ACM Transactions on Graphics; 2010; 29: 123.

[3] Golas A, Narain R, Curtis S, Lin MC. Hybrid Long-Range Collision Avoidance for Crowd Simulation. IEEE Trans Vis Comput Graph. 2014;20(7):1022-1034.

[4] Helbing D, Buzna L, Johansson A, Werner T. Self-organized pedestrian crowd dynamics: Experiments, simulations, and design solutions. Transportation Science. 2005;39(1):1-24.

[5] Moussaid M, Helbing D, Garnier S, Johansson A, Combe M, Theraulaz G. Experimental study of the behavioural mechanisms underlying self-organization in human crowds. Proc Biol Sci. 2009;276(1668):2755-2762. 